\title{
Smart Phone: An Embedded System for Universal Interactions *
}

\author{
Liviu Iftode, Cristian Borcea, Nishkam Ravi, Porlin Kang, and Peng Zhou \\ Department of Computer Science, Rutgers University, Piscataway, NJ 08854, USA \\ \{iftode, borcea, nravi, kangp, pzhou\}@ cs.rutgers.edu
}

\begin{abstract}
In this paper, we present a system architecture that allows users to interact with embedded systems located in their proximity using Smart Phones. We have identified four models of interaction between a Smart Phone and the surrounding environment: universal remote control, dual connectivity, gateway connectivity, and peer-to-peer. Although each of these models has different characteristics, our architecture provides a unique framework for all of the models. Central to our architecture are the hybrid communication capabilities incorporated in the Smart Phones. These phones have the unique feature of incorporating shortrange wireless connectivity (e.g., Bluetooth) and Internet connectivity (e.g., GPRS) in the same personal mobile device. This feature together with significant processing power and memory can turn a Smart Phone into the only mobile device that people will carry wherever they go.
\end{abstract}

\section{Introduction}

Recent advances in technology make it feasible to incorporate significant processing power in almost every device that we encounter in our daily life. These embedded systems are heterogeneous, distributed everywhere in the surrounding environment, and capable of communicating through wired or wireless interfaces. For a number of years, visionary papers $[21,18]$ have presented a picturesque computerized physical world with which we can potentially interact faster and in a simpler fashion.

People, however, are not yet taking advantage of this ubiquitous computing world. Despite all the computing power laying around, most of our daily interactions with the surrounding environment are still primitive and far from the ubiquitous computing vision. Our pockets and bags are still jammed with a bunch of keys for the doors we have to open/close daily (they did not change much since the Middle Ages), the car key or remote, access cards, credit cards,

* This work is supported in part by the NSF under the ITR Grant Number ANI-0121416 and money to pay for goods. Any of these forgotten at home can turn the day into a nightmare. If we travel, we also need maps and travel guides, coins to pay the parking in the city, and tickets to take the train or subway. In addition, we are always carrying our mobile phone, which for some mysterious reason is the least likely to be left at home. When we finally arrive home or at the hotel, we are "greeted" by several remote controls eager to test our intelligence. All these items are absolutely necessary for us to properly interact with our environment. The problem is that there are too many of them, they are sometimes heavy, and we will likely accumulate more and more of them as our life goes on, requiring much larger pockets.

For this problem, the community does not lack innovative solutions that address some of its aspects (e.g., wireless microservers [15], electronic payment methods [1,8], digital door keys [13]). What is missing is a simple, universal solution, which end-users are likely to accept easily. Ideally, we would like to have a single device that acts as both personal server [20] and personal assistant for remote interaction with embedded systems located in proximity of the user. This device should be programmable and support dynamic software extensions for interaction with newly encountered embedded systems (i.e., dynamically loading new interfaces). To simplify its acceptance by society, it should be a device that is already carried by people wherever they go.

We believe that Smart Phones are the devices that have the greatest chance of successfully becoming universal remote controls for people to interact with various devices from their surrounding environment; they will also replace all the different items we currently carry in our pockets. Smart Phone is an emerging mobile phone technology that supports Java program execution and provides both shortrange wireless connectivity (Bluetooth) and cellular network connectivity through which the Internet can be accessed.

In this paper, we present a system architecture that allows users to interact with embedded systems located in their proximity using a Smart Phone. We have identified four models of interaction between a Smart Phone and the surrounding environment: universal remote control, dual con- 
nectivity, gateway connectivity, and peer-to-peer. Although each of these models has different characteristics, our architecture provides a unique framework for all the models. Central to our architecture are the hybrid communication capabilities incorporated in the Smart Phones which allow them to interact with the close-by environment through short-range wireless networking and with the rest of the world through the Internet over cellular links. This feature together with significant processing power and memory can turn a Smart Phone into the long awaited universal personal assistant that can make our daily life much simpler.

The rest of this paper is organized as follows. Section 2 presents the enabling technologies for Smart Phones. Section 3 describes the four models of interaction between a Smart Phone and the embedded systems located within its proximity. Section 4 presents the unified software architecture for all the models. In section 5, we discuss the current status and future work. Section 6 presents the related work, and the paper concludes in Section 7.

\section{Smart Phones Technology}

With more than a billion mobile phones being carried around by consumers of all ages, the mobile phone has become the most pervasive pocket-carried device. We are beginning to see the introduction of Smart Phones, such as Sony Ericsson P800/P900 [9] and Motorola A760 [10] (Figure 1), as a result of the convergence of mobile phones and PDA devices. Unlike traditional mobile phones, which have limited processing power and act merely as "dumb" conduits for passing voice or data between the cellular network and end users, Smart Phones combine significant computing power with memory, short-range wireless interfaces (e.g., Bluetooth), Internet connectivity (over GPRS), and various input-output components (e.g., high-resolution color touch screens, digital cameras, and MP3 players).

Sony Ericsson P800/P900 runs Symbian OS [12], an operating system specifically designed for resource constrained devices such as mobile phones. It also comes equipped with two versions of Java technology: Personal Java [11] and J2ME CLDC/MIDP [2]. Additionally, it supports $\mathrm{C}++$ which provides low level access to the operating system and the Bluetooth driver. The phone has $16 \mathrm{MB}$ of internal memory and up to $128 \mathrm{MB}$ external flash memory. Motorola A760 has a Motorola i250 chip for communication, Intel's $200 \mathrm{MHz}$ PXA262 chip for computation, and $256 \mathrm{MB}$ of RAM memory. It runs a version of MontaVista Linux and comes with Java J2ME support [2].

Bluetooth [7] is a low-cost, low-power standard for wireless connectivity. Today, we can find Bluetooth chips embedded in PCs, laptops, digital cameras, GPS devices, Smart Phones, and a whole range of other elec-

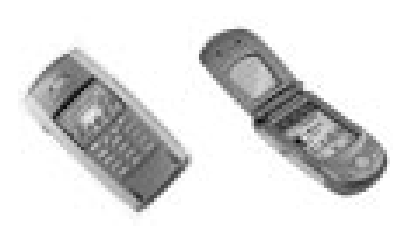

Figure 1. Example of Smart Phones: Sony Ericsson P800 (Left) and Motorola A760 (Right)

tronic devices. Bluetooth supports point-to-point and point-to-multipoint connections. We can actively connect a Bluetooth device to up to seven devices simultaneously. Together, they form an ad hoc network, called Piconet. Several piconets can be linked to form a Scatternet.

Another important development for the mobile phone technology is the introduction of General Packet Radio Service (GPRS) [3], a packet switching technology over the current GSM cellular networks. GPRS is offered as a nonvoice value-added service that allows data to be sent and received across GSM cellular networks at a rate of up to $171.2 \mathrm{kbps}$, and its goal is to supplement today's Circuit Switched Data and Short Message Service. GPRS offers an always-on service and supports Internet protocols.

\section{Smart Phone Interaction Models}

A Smart Phone can be used to interact with the surrounding environment in different ways. We have identified four interaction models: universal remote control, dual connectivity, gateway connectivity, and peer-to-peer. With these models, a Smart Phone can be used to execute applications from as simple as remotely adjusting various controls of home appliances or opening smart locks to complex applications such as automatically booking a cab or ordering/paying in a restaurant using an ad hoc network of mobile phones to connect to the cashier's computer.

\subsection{Universal Remote Control Model}

The Smart Phone can act as a universal remote control for interaction with embedded systems located in its proximity. To support proximity-aware interactions, both the Smart Phone and the embedded systems with which the user interacts must have short-range wireless communication capabilities. Figure 2 illustrates such interactions using Bluetooth. Due to its low-power, low-cost features, Bluetooth is the primary candidate for the short-range wireless technology that will enable proximity-aware communication. 


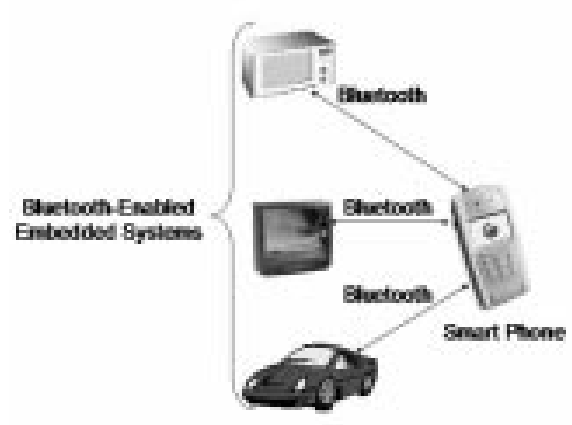

Figure 2. The Universal Remote Control Interaction Model

Since embedded systems with different functionalities can be scattered everywhere, a discovery protocol will allow Smart Phones to learn the identity and the description of the embedded systems located in their proximity. This protocol can work either automatically or on-demand, but the information about the devices currently located in user's proximity is displayed only upon user's request. Each embedded system should be able to provide its identity information (unique to a device or to a class of devices) and a description of its basic functionality in a human-understandable format.

This model works well as long as the user has the interfaces for interacting with the embedded systems preinstalled on the phone. An alternative, more flexible, solution is to define a protocol that allows a Smart Phone to learn the interfaces from the embedded systems themselves. The problem with this idea is that many embedded systems may not be powerful enough to run complex software that implements such protocols. In the following, we describe a second model of interaction that solves this problem.

\subsection{Dual Connectivity Model}

Central to our universal interaction architecture is the dual connectivity model which is based on the hybrid communication capabilities incorporated in the Smart Phones. They have the unique feature of incorporating both shortrange wireless connectivity (e.g., Bluetooth) and Internet connectivity (e.g., GPRS) in the same personal mobile device. With this model, the users can interact with the closeby environment using the short-range wireless connectivity and with the rest of the world using the Internet connectivity. Figure 3 illustrates the Dual Connectivity interaction model.

As a typical application, let us assume that a person has just bought an "intelligent" microwave oven equipped with a Bluetooth interface. This embedded system is very simple and is not capable of storing or transferring its inter-

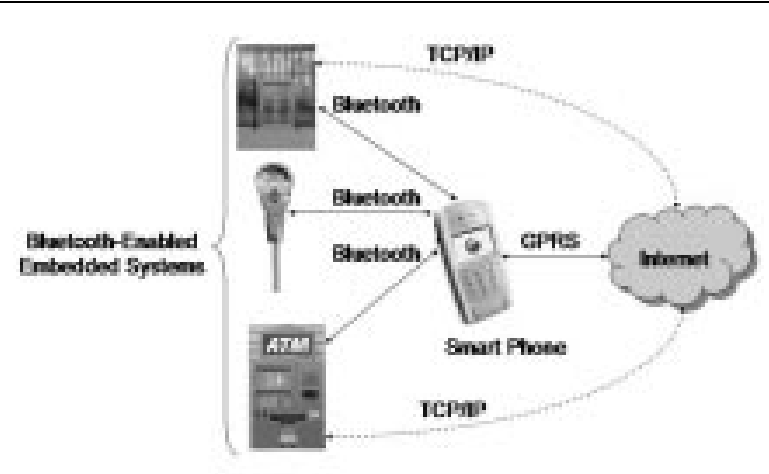

Figure 3. The Dual Connectivity Interaction Model

face to a Smart Phone. However, it is able to identify itself to Smart Phones. Using this information, the phones can connect to a server across the Internet (i.e., over GPRS) to download the code of the interface that will allow it to become a remote control for the microwave oven. The phone can also perform authentication over the Internet to ensure that the code is trusted. All further communication between this embedded system and the Smart Phone happens by executing the downloaded code. This code will display a panel that emulates the panel of the microwave on the phone's screen (i.e., it effectively transforms the phone into an intuitive microwave remote control).

Another typical application is opening/closing Smart Locks. We envision that the entry in certain buildings will soon be protected by Smart Locks (e.g., locks that are Bluetooth-enabled and can be opened using digital door keys). The dual connectivity model enables users carrying Smart Phones to open these locks in a secure manner. The Smart Phone can establish a connection with the lock, obtain the ID of the lock, and connect to an Internet server over GPRS to download the code that will be used for opening the lock (a digital door key can also be downloaded at the same time). The server hosting the interface and the keys for the Smart Lock maintains a list of people that are allowed to open the lock. The identity of the Smart Phone user (stored on the Smart Phone in the form of personal information) is piggybacked on the request submitted to the server. If the server finds that this user is allowed to open the lock, it responds with the code for the interface and the digital key.

The dual connectivity model can also be used to implement electronic payment applications similar to Millicent [1]. A client does not need to know about a vendor's embedded system in advance. The Smart Phone can authenticate the vendor using its Internet connection. The same connection can be used by the client to withdraw electronic currency from her bank and store it on the phone. Another 


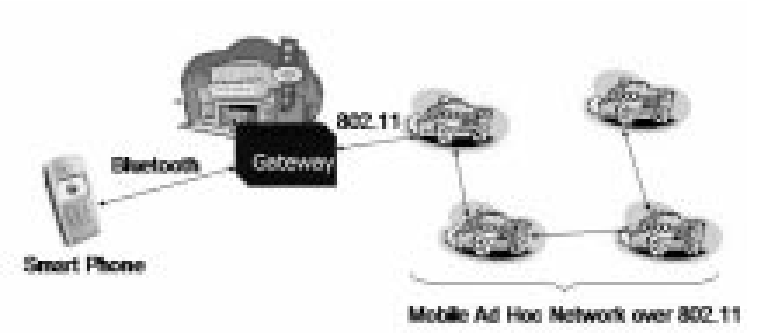

Figure 4. The Gateway Connectivity Interaction Model

option provided by the Smart Phone is to send some of the unused money back into the bank account (i.e., make a deposit each time the amount on the phone exceeds a certain limit). Potentially, the vendor's embedded system can also be connected to the Internet. For instance, this ability can be used to authenticate the client. Figure 3 presents a similar application that involves accessing an ATM using a Smart Phone.

\subsection{Gateway Connectivity Model}

Many pervasive applications assume wireless communication through the IEEE 802.11 family of protocols. These protocols allow for a significant increase in the communication distance and bandwidth compared to Bluetooth. Using these protocols, the communication range is $250 \mathrm{~m}$ or more, while Bluetooth reaches only $10 \mathrm{~m}$. The bandwidth is also larger, $11-54 \mathrm{Mbps}$ compared to less than $1 \mathrm{Mbps}$ for Bluetooth. Additionally, many routing protocols for mobile ad hoc networks based 802.11 already exist $[19,16]$. The disadvantage of 802.11 is that it consumes too much energy, and consequently, it drains out the mobile devices' batteries in a very short period of time. With the current state of the art, we do not expect to have 802.11 network interfaces embedded in Smart Phones or other resource constrained embedded systems that need to run on batteries for a significant period of time (e.g., several hours or even days).

More powerful systems, however, can take advantage of the 802.11 benefits and create mobile ad hoc networks. In such a situation, a user would like to access data and services provided by these networks from its Smart Phone. To succeed, a gateway device has to perform a change of protocol from Bluetooth to 802.11 and vice-versa. Many places in a city (e.g., stores, theaters, restaurants) can provide such gateway stations together with 802.11 hotspots.

Figure 4 illustrates this communication model and also presents an application that can be built on top of it. Let us assume a scenario where people want to book nearby cabs using their Smart Phones. Instead of calling a taxi company or "gesturing" to book a cab, a client can start an applica-

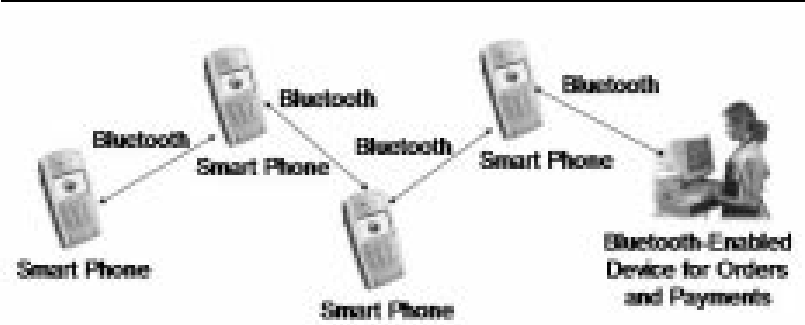

Figure 5. The Peer-to-Peer Interaction Model

tion on her Smart Phone that seamlessly achieves the same goal. Hence, the client is just one-click away from booking a cab. In this scenario, each cab is equipped with 802.11 wireless networking and GPS devices, and the entire booking process is completely decentralized. To join the mobile ad hoc network created by the cabs, a Smart Phone needs to connect to a gateway station that performs a translation of protocols from Bluetooth to 802.11 and vice-versa.

\subsection{Peer-to-Peer Model}

The Smart Phones can also communicate among themselves (or with other Bluetooth-enabled devices) in a multihop, peer-to-peer fashion, similar to mobile ad hoc networks. For instance, this model allows people to share music and pictures with others even if they are not in the proximity of each other. Figure 5 depicts yet another example of this model. A group of friends having dinner in a restaurant can use their Smart Phones to execute a program that shares the check. One phone initiates this process, an ad hoc network of Smart Phones is created, and finally the payment message arrives at the cashier.

\section{System Architecture}

Our system architecture for universal interaction consists of a common Smart Phone software architecture and an interaction protocol. This protocol allows Smart Phones to interact with the surrounding environment and the Internet. Figure 6 shows the Smart Phone software architecture. In the following, we briefly describe the components of the software architecture.

- Bluetooth Engine is responsible for communicating with the Bluetooth-enabled embedded systems. It is composed of sub-components for device discovery and sending/receiving data. The Bluetooth Engine is a layer above the Bluetooth stack and provides a convenient Java API for accessing the Bluetooth stack.

- Internet Access Module carries out the communication between the Smart Phone and various Internet 


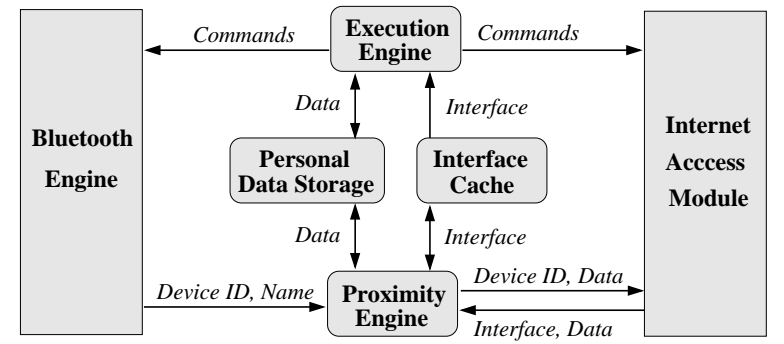

Figure 6. Smart Phone Software Architecture

servers. It provides a well-defined API that supports operations specific to our architecture (e.g., downloading an interface). The protocol of communication is HTTP on top of GPRS.

- Proximity Engine is responsible for discovering the embedded systems located within the Bluetooth communication range. Each time the user wants to interact with one of these systems, and an interface for this system is not available locally (i.e., a miss in the Interface Cache), the Proximity Engine is responsible from downloading such an interface. If the embedded system has enough computing power and memory, the interface can be downloaded directly from it. Otherwise, the Proximity Engine invokes the Internet Access Module to connect to a web server and download the interface. The downloaded interface is stored in the Interface Cache for later reuse. Once this is done, the Proximity Engine informs the Execution Engine to dispatch the downloaded interface for execution. All further communication between the Smart Phone and the embedded system happens as a result of executing this interface.

- Execution Engine is invoked by the Proximity Engine and is responsible for dispatching interface programs for execution over the Java virtual machine. These programs interact with the Bluetooth Engine to communicate with the embedded systems or with other Smart Phones (as described in Section 3.4). They may also interact with the Internet Access Module to communicate with Internet servers. For instance, the interface programs may need to contact a server for securityrelated actions or to download necessary data in case of a miss in the Personal Data Storage.

- Interface Cache stores the code of the downloaded interfaces. This cache avoids downloading an interface every time it is needed. An interface can be shared by an entire class of embedded systems (e.g., Smart
Locks, or Microwaves). Every interface has an ID (which can be the ID of the embedded system or the class of embedded systems it is associated with). This ID helps in recognizing the cached interface each time it needs to be looked up in the cache. Additionally, each interface has an associated access handler that is executed before any subsequent execution of the interface. This handler may define the time period for which the interface should be cached, how and when the interface can be reused, or the permissions to access local resources. The user can set the access handler's parameters before the first execution of the interface.

- Personal Data Storage acts as a cache for "active data", similar to Active Cache [14]. It stores data that needs to be used during the interactions with various embedded systems. Examples of such data include digital door keys and electronic cash. Each data item stored in this cache has three associated handlers: access handler, miss handler, and eviction handler. Each time an interface needs some data, it checks the Personal Data Storage. If the data is available locally (i.e., hit), the access handler is executed, and the program goes ahead. For instance, the access handler may check if this data can be shared among different interfaces. If the data is not available locally (i.e., miss), the miss handler instructs the Internet Access Module to download the data from the corresponding Internet server. The eviction handler defines the actions to be taken when data is evicted from the cache. For instance, electronic cash can be sent back to the bank at eviction time.

Figure 7 shows the interaction protocol that takes place when a Smart Phone needs to interact with an embedded system. We consider that any embedded system is registered with a trusted web server (this web server can be physically distributed on multiple computers). At registration, the web server assigns a unique ID and a URL to the device. All the information necessary to interact with the device along with a user interface is stored at that URL. This URL may be common for an entire class of embedded systems.

The user invokes the Proximity Engine each time she needs to interact with a device located in the proximity. Once the embedded systems in the proximity have been identified, the user can choose the one she wants to interact with. Consequently, a request is sent to the embedded system to provide its ID and URL. Upon receiving the ID and URL of the embedded system, the Smart Phone executes the access control handler, and then, loads and executes the interface. In case of a miss in the Interface Cache, the interface needs to be downloaded on the phone either from the web server or from the embedded system itself. An inter- 


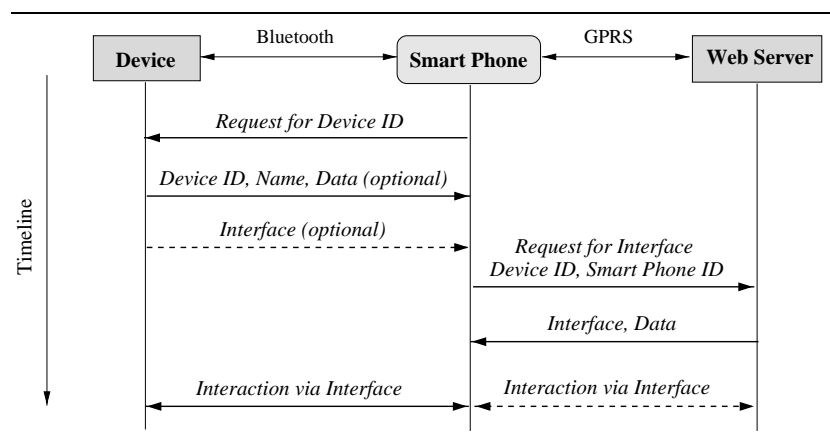

Figure 7. Smart Phone Interaction Protocol

face downloaded from an embedded system is untrusted and is not allowed to access local resources (i.e., this is a sandbox model of execution, where the interface can only execute safe instructions on the phone). The interfaces downloaded from the web server are trusted; they are assumed to be verified before being distributed by the server.

Each time a Smart Phone requests an interface from the web server, it has to send the interface ID and the URL provided by the embedded system. It also sends its ID (stored in the Personal Data Storage). The permission to download an interface is subject to access control enforced based on the Smart Phone ID and, potentially, other credentials presented by the user. Once the access is granted, the web server responds with the interface code.

\section{Status and Future Work}

In this section, we briefly outline the current status and several open issues that we have to overcome in order to implement our system architecture. We are in the process of building the system architecture on top of Ericsson's P800/900 phones. Our first step consists of implementing the basic architecture for the universal remote control interaction model. The architecture components to be developed for this model are the Bluetooth Engine and Proximity Engine along with a simple Execution engine over Java.

We have partially implemented the Bluetooth Engine and have written and tested a few sample programs to test the feasibility of connecting a phone to another phone or to a Bluetooth-enabled laptop. Besides directly connecting to Bluetooth-enabled devices, a phone can also connect to a LAN. We are in the process of investigating the feasibility of using the Bluetooth LAN profile to connect the phone to a LAN through a Bluetooth access point.

Until recently, the commercially available Bluetooth chips have been working well for one-hop communication, but their scatternet capabilities have not been mature enough to support multi-hop communica- tion as needed in our peer-to-peer interaction model. Currently, there are products [4], however, whose scatternet capabilities have been successfully tested. We envision that multi-hop communication in ad hoc networks will take place either over Bluetooth or over 802.11 depending on the trade-offs between the battery power consumption and communication range. Our system architecture supports both situations through the peer-to-peer model and the gateway model, respectively.

To connect a Smart Phone to the Internet over GPRS, we can use HTTP or TCP. A decision regarding the protocol used for Internet access needs to consider the trade-offs between the simplicity provided by HTTP and the flexibility and efficiency provided by TCP.

Although our architecture provides a level of security by obtaining interface code and confidential data from a trusted web server, many issues related to security and privacy still need to be addressed. For instance, we need to investigate different lightweight encryption algorithms that work on resource constrained devices to counter eavesdropping without a serious overhead. So far we have assumed that the personal information of the user, including confidential data, would be stored on the Smart Phone. In such a situation, losing the Smart Phone could pose a serious security threat to the owner. The data stored on the phone should be made inaccessible to anyone but the phone owner. A simple password scheme is insufficient because entering a password every time confidential data is accessed could be a major turn off for the users. We plan to investigate both software protection mechanisms and hardware solutions (e.g., biometric security using fingerprint recognition [5]).

\section{Related Work}

Personal Server [20] is a small-size mobile device that stores user's data on a removable Compact Flash and wirelessly utilizes any I/O interface available in its proximity (e.g., display, keyboard). Its main goal is to provide the user with a virtual personal computer wherever the user goes. Our goal is to provide a simple method of interaction with systems embedded in the surrounding environment. Unlike Personal Server which cannot connect directly to the Internet, Smart Phones do not have to carry every possible data or code that the user may need; they can download ondemand data and code for interfaces from the Internet.

CoolTown [17] proposes web presence as a basis for bridging the physical world with the World Wide Web. For example, entities in the physical world are embedded with URL-emitting devices (beacons) which advertise the URL for the corresponding entities. Our model is more flexible as we allow code and data to be downloaded to mobile devices, either from the physical environment via short-range 
wireless connection, or from the Internet via the GPRS connection.

Microservers [15] share one of our goals of turning a handheld device into a universal remote control. Their approach consists of embedding web servers in Bluetoothenabled devices and using WAP over Bluetooth to communicate between the handheld and these devices. Our approach is more practical since it does not require any complex software to be installed on resource constrained embedded systems. Additionally, it covers other interaction models besides the universal remote control model (e.g., gateway model, peer-to-peer model).

Jini [6] is a system designed to deal with resource discovery and interaction in a new environment. When a service joins a network of Jini-enabled services, it advertises itself by publishing an object that implements the service API. The client finds services by looking for an object that supports this API. When it gets the service's published object, it downloads any code it needs in order to talk to the service (via RMI, CORBA, XML, or any private protocols). We share a few design principles with Jini, but unlike Jini, our architecture does not require an infrastructure, and therefore, it is more suitable for ubiquitous computing environments.

The idea of using digital door keys to unlock doors has already been proposed [13] as an addition to a Personal Server. However, the issue of digital door key distribution from the external authority to the Personal Servers is not addressed. Our work uses the Smart Phone as an incarnation of a Personal Server and also addresses the issue of secure key distribution. More generally, our system architecture provides a general framework that can be used to implement any application that needs to interact with wireless embedded systems.

\section{Conclusions}

In this paper, we have argued for turning the Smart Phone into the only device that people carry in their pockets wherever they go. The Smart Phone can be used as both personal server that stores or downloads data that its user needs and personal assistant for remote interaction with embedded systems located in the user's proximity. To achieve this vision, we have presented a unified system architecture for different models of interaction between a Smart Phone and the surrounding environment. Central to this universal interaction architecture is the dual connectivity feature of Smart Phones, which allows them to interact with the close-by environment through short-range wireless networking and with the rest of the world through the Internet over cellular links.

\section{References}

[1] The Millicent Protocol for Inexpensive Electronic Commerce. http://www.w3.org/Conferences/WWW4 /Papers/246/.

[2] MIDP Profile. http://wireless.java.sun.com/midp/.

[3] General Packet Radio Service (GPRS). http://www.gsmworld.com/technology/gprs/intro.shtml.

[4] Zeevo Bluetooth. http://www.azzurri.com/new_htm/zeevo.htm.

[5] HP iPAQ 5400. http://welcome.hp.com/country/us/en /prodserv/handheld.html.

[6] Jini Network Technology. http://wwws.sun.com/software/jini.

[7] Bluetooth. https://www.bluetooth.org/.

[8] Digicash. http://www.digicash.com.

[9] Ericsson P800. http://www.sonyericsson.com/P800/.

[10] Motorola A760. http://motoinfo.motorola.com/motoinfo /products.asp?product=A760\&y=2003.

[11] PersonalJava. http://java.sun.com/j2me/.

[12] Symbian OS. http://www.symbian.com/.

[13] A. Beaufour and P. Bonnet. Personal Servers as Digital Keys. In Proceedings of the 2nd IEEE International Conference on Pervasive Computing and Communications (PerCom 2004). To Appear, 2004.

[14] P. Cao, J. Zhang, and K. Beach. Active Cache: Caching Dynamic Contents on the Web . In Proceedings of IFIP International Conference on Distributed Systems Platforms and Open Distributed Processing (Middleware 1998), pages 373-388, 1998.

[15] S. Hartwig, J.-P. Stromann, and P. Resch. Wireless Microservers. Pervasive Computing, 1(2):58-66, April-June 2002.

[16] D. Johnson and D. Maltz. Dynamic Source Routing in Ad Hoc Wireless Networks. T. Imielinski and H. Korth, (Eds.). Kluwer Academic Publishers, 1996.

[17] T. Kindberg, J. Barton, J. Morgan, G. Becker, D. Caswell, P. Debaty, G. Gopal, M. Frid, V. Krishnan, H. Morris, J. Schettino, B. Serra, and M. Spasojevic. People, places, things: Web presence for the real world. In Proceedings of the 3rd IEEE Workshop on Mobile Computing Systems and Applications, pages 19-28, 2000.

[18] M. Satyanarayanan. Pervasive Computing: Vision and Challenges. IEEE Personal Communications, August 2001.

[19] C. Perkins and E. Royer. Ad Hoc On Demand Distance Vector Routing. In Proceedings of the 2nd IEEE Workshop on Mobile Computing Systems and Applications (WMCSA 1999), pages 90-100, New Orleans, LA, February 1999.

[20] R. Want, T. Pering, G. Dianneels, M. Kumar, M. Sundar, and J. Light. The Personal Server: Changing the Way We Think about Ubiquitous Computing. In Proceedings of 4th International Conference on Ubiquitous Computing (Ubicomp 2002), pages 194-209. Springer LNCS, September 2003.

[21] M. Weiser. The computer for the twenty-first century. Scientific American, September 1991. 